\title{
Manejo actual de las metástasis vertebrales: un trabajo en equipo
}

\section{Current Management of Vertebral Metastases: Teamwork}

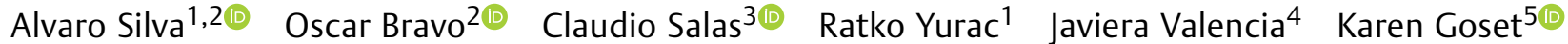 \\ Hans Harbst ${ }^{5}$ Andrés Córdova ${ }^{5}$
}

1 Departamento de Traumatología, Unidad de Columna, Clínica Alemana de Santiago, Universidad del Desarrollo, Santiago, Chile Dirección para correspondencia Dr. Alvaro Silva, MD, Clínica Alemana de Santiago, Av. Vitacura 5.951, Vitacura, Santiago, Chile

2 Departamento de Traumatología, Equipo de Columna, Hospital FACh, Santiago, Chile

${ }^{3}$ Departamento de Oncología, Servicio de Oncología, Clínica Alemana de Santiago, Universidad del Desarrollo, Santiago, Chile

${ }^{4}$ Universidad de los Andes, Santiago, Chile

${ }^{5}$ Departamento de Oncología, Servicio de Radioterapia, Clínica

Alemana de Santiago, Universidad del Desarrollo, Santiago, Chile (e-mail: asilvag@alemana.cl).

Rev Chil Ortop Traumatol 2021;62(2):e136-e142.

\section{Resumen \\ Palabras Clave \\ - metástasis vertebrales \\ - radiocirugía vertebral \\ - cirugía de separación \\ - revisión}

La enfermedad metastásica vertebral es frecuente en los pacientes con cáncer avanzado, y conlleva a complicaciones inherentes a su progresión, como lo son la fractura patológica vertebral y la compresión neural metastásica. Se realizó una revisión de los aspectos terapéuticos actuales del manejo de la progresión y de las complicaciones de la enfermedad metastásica vertebral, enfatizando su enfrentamiento sistémico y personalizado. Nuestro objetivo principal es proporcionar información sobre el tratamiento actual de esta afección y la utilidad del manejo sistémico y multidisciplinario.

Metastatic spinal disease is common in patients with advanced cancer, and leads to complications inherent in its progression, such as pathological vertebral fracture and metastatic neural compression. A review of the current therapeutic aspects regarding the progression and complications of metastatic spinal disease was performed, highlighting its systemic, personalized approach. Our aim is to provide information about the current treatment of this condition and the usefulness of its systemic and multidisciplinary management. recibido

05 de julio de 2020

aceptado

31 de marzo de 2021
DOI https://doi.org/

$10.1055 / \mathrm{s}-0041-1735295$ ISSN $0716-4548$. (c) 2021. Sociedad Chilena de Ortopedia y Traumatologia. All rights reserved.

This is an open access article published by Thieme under the terms of the Creative Commons Attribution-NonDerivative-NonCommercial-License, permitting copying and reproduction so long as the original work is given appropriate credit. Contents may not be used for commercial purposes, or adapted, remixed, transformed or built upon. (https://creativecommons.org/ licenses/by-nc-nd/4.0/)

Thieme Revinter Publicações Ltda., Rua do Matoso 170, Rio de Janeiro, RJ, CEP 20270-135, Brazil 


\section{Generalidades}

El 40\% de los pacientes afectos de cáncer cursan durante la evolución de su enfermedad con metástasis vertebrales. El 80\% de estas metástasis se originan de tumores de la mama, de tumores de la próstata, y de neoplasias del pulmón. La vía de diseminación es mayoritariamente hematógena: arterial para los platillos vertebrales; por reflujo venoso a través de los plexos de Batson para el cuerpo vertebral; a través de mecanismos de diseminación intravertebral específicos de los tejidos y por circulación intravertebral. ${ }^{1}$ El $75 \%$ de las metástasis vertebrales se ubica en columna tóracolumbar. La localización de las metástasis es en un $80 \%$ en la vértebra, principalmente en la zona de unión del pedículo con el cuerpo vertebral; en un $15 \%$, se ubican en tejidos perivertebrales; $y$, en sólo un $5 \%$ de los pacientes, la metástasis se desarrolla dentro del espacio epidural. La localización de las metástasis vertebrales generalmente es múltiple, mientras que sólo un $10 \%$ de ellas se ubican aisladas. ${ }^{2}$

Durante la evolución de los pacientes con cáncer, un 30\% a $70 \%$ presentan metástasis en estudios post mortem, y alrededor del $25 \%$ presentan síntomas. En estos pacientes sintomáticos, lo más frecuente es el dolor en el 75\%, fractura vertebral patológica en el 10\%, hipercalcemia en el $10 \%$, y lesión medular o radicular en el $5 \%{ }^{3}$

En la mayoría de los pacientes, el objetivo del tratamiento de las metástasis vertebrales es paliativo, orientado a los síntomas derivados de la invasión tumoral de la vértebra y del espacio epidural, manejar el dolor, mejorar o preservar la función neurológica, prevenir o tratar una fractura patológica, y obtener el control local de la enfermedad. Todo esto con el objetivo de mejorar la calidad de vida del último período de vida del paciente, siempre con el cuidado de minimizar los efectos adversos o complicaciones del tratamiento. ${ }^{4}$

El objetivo de este artículo es revisar la literatura y exponer nuestra conducta frente al tratamiento de las metástasis vertebrales desde un punto de vista integral entre el oncólogo, el radioterapeuta, y el cirujano de columna.

Las herramientas terapéuticas disponibles para el tratamiento de las complicaciones locales de las metástasis vertebrales son: el tratamiento médico (corticoterapia, quimioterapia, hormoterapia, inmunoterapia, y uso de inhibidores de osteoclastos), la radioterapia, y la cirugía.

\section{El tratamiento médico: aumento de la sobrevida y contención de las metástasis}

El desarrollo de técnicas de tamizaje ha permitido una importante disminución de los principales tumores que afectan a la población mundial, como los de mama, pulmón y próstata. ${ }^{5}$

La evolución y progreso de las terapias farmacológicas y biológicas han permitido un avance sostenido en la curación y, sobretodo, en la prolongación de las expectativas de vida de los pacientes con cánceres avanzados.

En relación al cáncer de mama, el reconocimiento de distintos subtipos moleculares con un comportamiento clínico diferente $e^{6,7}$ y, por otro lado, el descubrimiento en los años 1980 de una proteína de transmembrana denominada receptor 2 del factor de crecimiento epidérmico humano (human epidermal growth factor receptor 2, HER2, en inglés) ${ }^{8}$ dieron origen a una revolución en el desarrollo de la biotecnología, y permitieron la aplicación clínica de anticuerpos monoclonales como el trastuzumab, que ha impactado favorablemente tanto en etapas tempranas como tardías, aumentando las tasas de curación y de sobrevida. ${ }^{9,10}$ Posteriormente, la incorporación de otros fármacos anti-HER 2, como pertuzumab, ${ }^{11}$ trastuzumab emtansine (TDM- 1$)^{12}$ y lapatinib, también ha aportado al cuidado de las pacientes en etapas avanzadas. ${ }^{13} \mathrm{La}$ aplicación de distintos tipos de hormonoterapia en pacientes que expresan receptores hormonales ha ampliado la posibilidad de curación en etapas tempranas, ${ }^{14}$ como también, la prolongación de sobrevida en etapas avanzadas, ${ }^{15} \mathrm{y}$, más recientemente, la incorporación de los inhibidores de quinasa dependiente de ciclina, como el palbociclib, ha mejorado las tasas de respuesta y sobrevida libre de progresión. ${ }^{16}$

El cáncer de pulmón es la principal causa de mortalidad por cáncer en el mundo, ${ }^{17}$ por lo que se han hecho esfuerzos por mejorar las combinaciones de quimioterapia citotóxica. ${ }^{18} \mathrm{Se}$ ha desarrollado una nueva clase de medicamentos, los inhibidores de la tirosina kinasa, que interfieren en las vías de señalización en un punto específico, y que han logrado mejorar la sobrevida libre de progresión en los pacientes con mutaciones especificas. ${ }^{19}$ La innovación más reciente en esta área ha sido el desarrollo de la inmunoterapia, que ha permitido mejorar la sobrevida de los pacientes con cáncer de pulmón, tanto la progresión del tumor primario como la de las metástasis. ${ }^{20-23}$

El cáncer de próstata es un cáncer frecuente, que, en etapas avanzadas, se asocia con una alta frecuencia de metástasis óseas, las que pueden ser controladas mediante hormonoterapia y quimioterapia. Históricamente, la primera intervención hormonal consistió en la castración quirúrgica. ${ }^{24}$ Posteriormente, se implementó la castración química con análogos de hormona liberadora de hormona luteinizante (luteinizing hormone-releasing hormone, LH-RH, en inglés); ${ }^{25} \mathrm{y}$, luego, la combinación de éstos con antiandrógenos, llamada bloqueo androgénico completo. ${ }^{26}$ Finalmente, nuevos antiandrógenos, como enzalutamida ${ }^{27}$ o abiraterona. ${ }^{28}$ Otras nuevas herramientas disponibles, como la quimioterapia con docetaxel y cabazitaxel, han mejorado de manera significativa la sobrevida global. ${ }^{29-32}$

El desarrollo de estas nuevas terapias oncológicas ha posibilitado la prolongación significativa de la sobrevida de los pacientes, y permitido que los pacientes vivan en buenas condiciones generales con un cáncer avanzado, pero que también tengan mayor oportunidad de presentar metástasis vertebrales y sus complicaciones, como dolor, fractura vertebral, y/o compromiso neurológico. ${ }^{33}$

\section{Tratamiento preventivo de las complicaciones de las metástasis vertebrales}

La administración de inhibidores de los osteoclastos, como los bifosfonatos, o inhibidores del ligando del receptor 
activador del factor nuclear kappa B (receptor activator of nuclear factor kappa-B ligand, RANKL, en inglés), como denosumab, ha demostrado un rol significativo en la disminución de eventos óseos relacionados a metástasis, como fractura vertebral patológica, necesidad de cirugía sobre hueso metastásico, o eventos de compresión mieloradicular. Sus beneficios superan con creces las potenciales complicaciones, como la necrosis ósea mandibular y las fracturas atípicas. El pamidronato fue uno de los primeros bifosfonatos endovenosos que demostró una reducción significativa de los eventos esqueléticos y un retardo del primer evento óseo comparado con un placebo. ${ }^{34}$ Posteriormente, se demostró la superioridad del ácido zolendrónico en comparación con el pamidronato. ${ }^{35}$ Finalmente, una nueva familia terapéutica, los inhibidores RANKL (denosumab), demostraró una superioridad de hasta un $18 \%$ sobre el ácido zolendrónico. ${ }^{36}$

La selección del tratamiento antiresortivo preventivo de eventos metastásicos va a depender de aspectos clínicos del paciente, como la extensión ósea de la enfermedad y la función renal. En paciente con función renal normal, nosotros utilizamos ácido zolendrónico, y en paciente con función renal deteriorada o con compromiso óseo muy extenso, utilizamos denosumab.

El límite de las terapias preventivas es cuando las metástasis vertebrales se vuelven sintomáticas por la aparición de dolor o por fractura patológica. En esta etapa, el uso de los inhibidores osteoclásticos no ha demostrado disminuir la sintomatología dolorosa, y menos la compresión de estructuras neurales. Si bien se deben seguir administrando para disminuir el riesgo de otras lesiones óseas que estén en un estadio menor, una vez que una
Tabla 1 Marco de decisión neurológica, oncológica, mecánica, y sistémica (NOMS) $)^{33}$

\begin{tabular}{|l|l|}
\hline $\mathrm{N}$ & Estatus neurológico \\
\hline $\mathrm{O}$ & Consideraciones oncológicas \\
\hline $\mathrm{M}$ & Estabilidad mecánica \\
\hline $\mathrm{S}$ & Enfermedad sistémica $^{\mathrm{a}}$ \\
\hline
\end{tabular}

Nota: ${ }^{a}$ Status general - pronóstico.

metástasis vertebral se complica y se vuelve sintomática, va a requerir de un tratamiento específico, ya sea: radioterapia, cirugía, o su combinación. ${ }^{37}$

\section{Categorización de los pacientes con metástasis vertebrales}

En la evaluación, categorización y definición de una conducta terapéutica para tratar a los pacientes con metástasis vertebrales, nosotros hemos incorporado el marco de decisión neurológica, oncológica, mecánica, y sistémica (neurologic, oncologic, mechanical, and systemic, NOMS, en inglés $)^{33}$ ( Tabla 1). Este sistema considera la evaluación del paciente con metástasis vertebrales de una manera integral, tomando en cuenta: el estatus neurológico $(\mathrm{N})$, que tiene que ver con el grado de compresión epidural del tumor sobre las estructuras neurales y el consiguiente compromiso neurológico $^{38}$ (- Figura 1); las consideraciones oncológicas (O) de la neoplasia, que tienen que ver con la biología del tumor y su radiosensibilidad (-Tabla 2); la estabilidad mecánica $(\mathrm{M})$ de la columna con una metástasis vertebral,
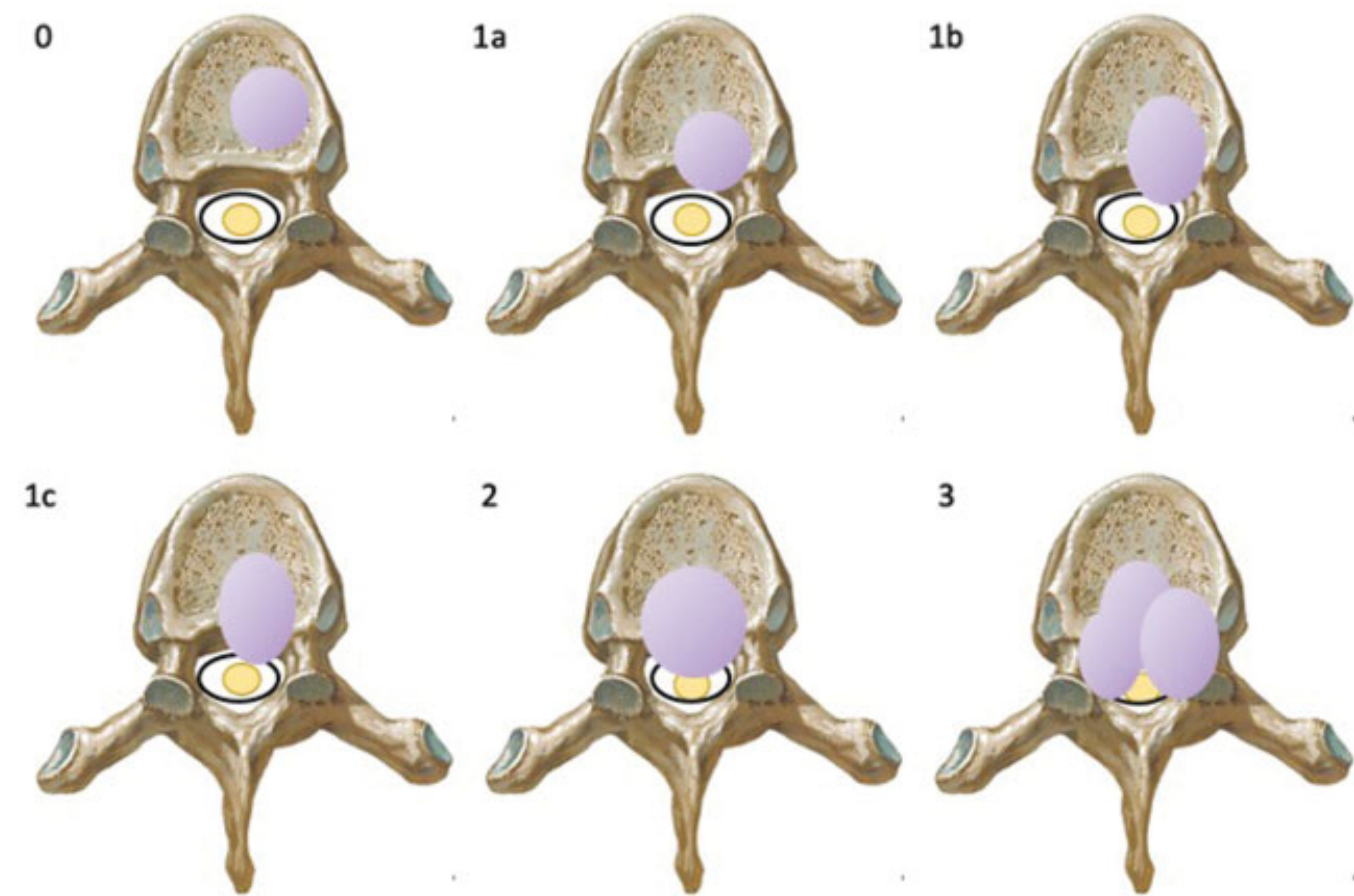

Fig. 1 Escala de Compresión Epidural de Médula Espinal de seis grados. ${ }^{38}$ 0: lesión confinada al cuerpo vertebral; 1a: invasión del espacio epidural sin deformación del saco dural; 1 b: deformación del saco dural sin contactar la médula; 1 c: contacta la médula, pero no la comprime; 2 : compresión medular con visualización de líquido cefalorraquídeo; y 3: compresión medular sin visualización de líquido cefalorraquídeo 
Tabla 2 Sensibilidad a la irradiación del tumor ${ }^{33}$

\begin{tabular}{|l|l|}
\hline Radiosensible $^{\mathbf{a}}$ & Radiorresistente $^{\mathbf{a}}$ \\
\hline Lifoma & Renal \\
\hline Mieloma multiple & Tiroides \\
\hline Seminoma & Hepatocelular \\
\hline Mama & Gastrointestinal \\
\hline Próstata & $\begin{array}{l}\text { Pulmón (células no } \\
\text { pequeñas) }\end{array}$ \\
\hline Ovario & Sarcoma \\
\hline Carcinoma neuroendocrino & Melanoma \\
\hline
\end{tabular}

Nota: ${ }^{\mathrm{T} T u m o r}$ tratado con radioterapia convencional.

en la que utilizamos el sistema de clasificación de estabilidad mecánica Spine Instability Neoplastic Score (SINS) ${ }^{39}$ (-Tabla 3) y su consiguiente riesgo de fractura, dolor y/o compresión neural; y el estado médico general o sistémico (S), del paciente que orientan a su potencial sobrevida.

Así, siguiendo este sistema de evaluación, podemos clasificar a cada paciente con una metástasis vertebral sintomática e indicarles tratamiento ajustado.

\section{Radioterapia}

La indicación ideal de radioterapia como tratamiento único es para casos de metástasis vertebral que produce dolor de reposo con preservación de la estabilidad mecánica de la columna a pesar de la invasión tumoral.

Actualmente, se dispone de varias técnicas de radioterapia externa. Dependiendo del objetivo de la terapia, de los volúmenes tumorales a cubrir, y del origen tumoral, se pueden definir diferentes esquemas de fraccionamiento de la dosis. Para paliar el dolor, en pacientes muy sintomáticos con expectativa de vida corta, se puede administrar una fracción única de 8 Gy. Para un paciente con mejores expectativas de vida, se pueden utilizar esquemas más convencionales de radioterapia convencional, como 30 Gy fraccionada en 10 sesiones. Estos tratamientos permiten controlar total o parcialmente el dolor en el $80 \%$ de los pacientes, en algunos casos durante muchos años.

Dependiendo de la cercanía a órganos especialmente sensibles, en casos de reirradiación o en aquellos tumores radiorresistentes, se pueden usar esquemas de dosis altas en pocas fracciones. En estos casos es ideal utilizar técnicas de radioterapia estereotáxica guiada por imágenes (image-guided stereotactic radiotherapy techniques, IGRTs, en inglés), que permite minimizar los márgenes de irradiación requeridos entre el tejido tumoral y los tejidos sanos circundantes, especialmente la médula espinal, disminuyendo así las complicaciones, como la mielopatía por radiación.

El disponer de estas nuevas técnicas ha permitido aumentar significativamente la dosis total administrada en algunos casos especiales, disminuyendo los casos de "radiorresistencia", como en los tumores de origen renal. ${ }^{40}$ Sin embargo, es importante tener en cuenta que estas
Tabla 3 Criterios del Spinal Instability Neoplastic Score (SINS) ${ }^{39}$

\begin{tabular}{|c|c|}
\hline Elementos SINS & Puntaje $^{a}$ \\
\hline \multicolumn{2}{|l|}{ Localización } \\
\hline $\begin{array}{l}\text { En la unión (occipital-C2, C7-T2, } \\
\text { T11-L1, L5-S1) }\end{array}$ & 3 \\
\hline Columna móvil (C3-C6, L2-L4) & 2 \\
\hline Semirrígida (T3-T10) & 1 \\
\hline Rígida (S2-S5) & 0 \\
\hline \multicolumn{2}{|l|}{ Dolor } \\
\hline Mecánico & 3 \\
\hline Ocasional y no mécanico & 1 \\
\hline Sin dolor & 0 \\
\hline \multicolumn{2}{|l|}{ Tipo de lesión ósea } \\
\hline Lítica & 2 \\
\hline Mixta (lítica y blástica) & 1 \\
\hline Blástica & 0 \\
\hline \multicolumn{2}{|l|}{ Alineación radiográfica de la columna } \\
\hline Subluxación/Traslación & 4 \\
\hline Cifosis/Escoliosis (de novo) & 2 \\
\hline Alineación normal & 0 \\
\hline \multicolumn{2}{|l|}{ Colapso o compromiso del cuerpo vertebral } \\
\hline$>50 \%$ & 3 \\
\hline$<50 \%$ & 2 \\
\hline $\begin{array}{l}\text { No hay colapso, pero con }>50 \% \text { del } \\
\text { cuerpo comprometido }\end{array}$ & 1 \\
\hline Ninguno de los anteriores & 0 \\
\hline \multicolumn{2}{|l|}{$\begin{array}{l}\text { Compromiso de elementos posterolaterales } \\
\text { (fractura o lesión tumoral) } \\
\text { (fula }\end{array}$} \\
\hline Bilateral & 3 \\
\hline Unilateral & 1 \\
\hline Ninguno de los anteriores & 0 \\
\hline
\end{tabular}

Notas: ${ }^{a}$ Sumatoria de los puntajes: 0 a 6, estable; 7 a 12, inestabilidad indeterminada (posiblemente inminente); y 13 a 18, inestable. ${ }^{b}$ Faceta, pedículo, o articulación costovertebral.

técnicas aumentan considerablemente el costo de las terapias, por lo que su uso debe ser racional.

Se debe tener presente que hasta un 38\% de estos pacientes que son sometidos a radioterapia pueden evolucionar al colapso, dolor y/o compresión neural en las ocho a diez semanas después de la irradiación, por lo que se recomienda hacer un seguimiento radiológico y clínico para pesquisar estas complicaciones. ${ }^{41}$

Con la excepción de la ubicación vertebral de linfomas o mielomas, ambas neoplasias muy sensibles a corticoides y radioterapia, el uso exclusivo de irradiación como tratamiento del compromiso neurológico por invasión tumoral epidural tiene un rendimiento significativamente menor de recuperación neurológica y mayor riesgo de complicaciones locales, por lo que, en estos pacientes, si su condición médica lo 

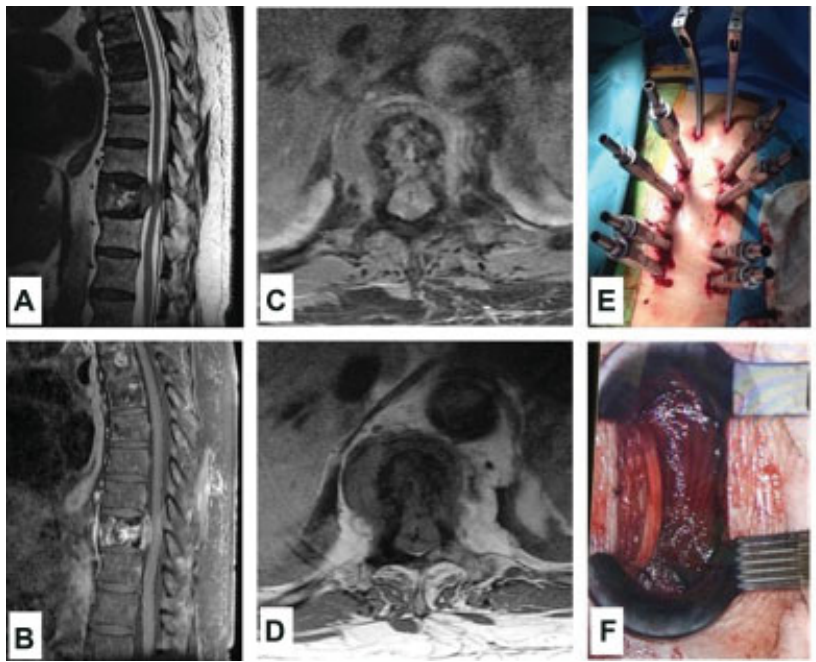
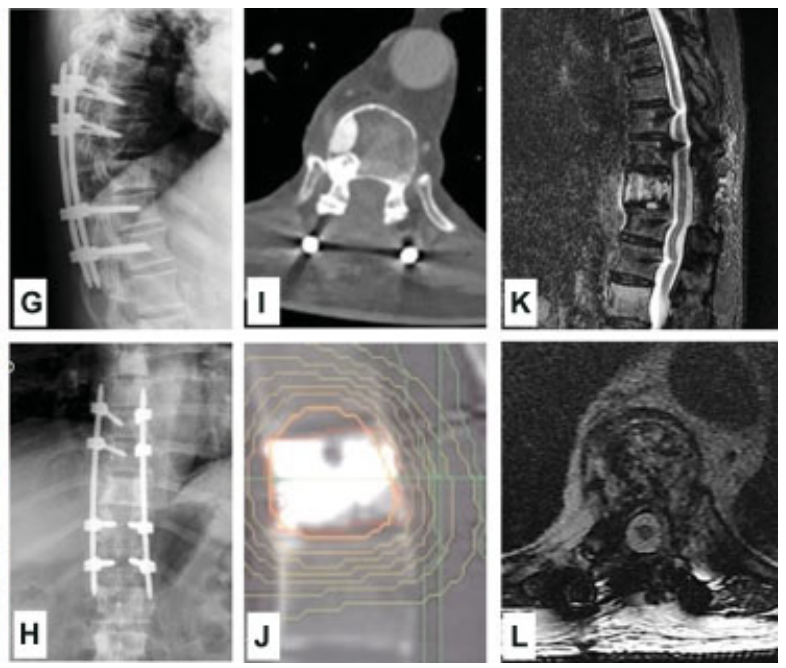

Fig. 2 Cirugía de separación tumoral y radioterapia estereotáxica postoperatoria. Hombre de 74 años con cáncer de próstata. Luego de ocho años de tratamiento de bloqueo hormonal, inicia cuadro progresivo de dolor dorsal mecánico y paraparesia. (A-D) Estudio de resonancia magnética (RM) de columna vertebral que evidencia metástasis vertebral de T10 con compresión medular grado 3. (E) Cirugía de fijación percutánea por vía posterior T8-T12. (F) Descompresión de canal raquídeo, resecando masa tumoral epidural. (G-H) Radiografía postoperatoria de columna que muestra el correcto posicionamiento de los implantes y alineamiento espinal. (I) Tomografía computarizada (TC) de columna de control postoperatorio de descompresión. (J) Planificación de técnicas de radioterapia estereotáxica guiada por imágenes (image-guided stereotactic radiotherapy techniques, IGRTs, en inglés). (K-L) Resonancia magnética de columna de control postradioterapia. Paciente con sobrevida actualizada de veinte meses, con recuperación de paraparesia.

permite, la recomendación de la literatura es que la cirugía de descompresión tumoral y estabilización quirúrgica se realice antes que la radioterapia. ${ }^{42}$

\section{Tratamiento quirúrgico}

La cirugía es la última herramienta para utilizar en el tratamiento de una metástasis vertebral sintomática. La indicación de cirugía se limita al tratamiento del dolor mecánico producido por una fractura patológica y a la compresión de estructuras neurales.

En los casos de pacientes con dolor mecánico por fractura patológica, pero que no tienen compromiso neurológico, y que, según la escala de estabilidad SINS, tienen un puntaje intermedio, es decir, lesiones potencialmente inestables en lo mecánico, utilizamos técnicas de aumentación vertebral, como la vertebroplastía o la cifoplastía. Estos son procedimientos quirúrgicos percutáneos de muy poca agresión y con una probada eficacia en el control del dolor en fracturas vertebrales patológicas. Consisten en la inyección intravertebral de cemento acrílico (polimetilmetacrilato) que se distribuye dentro de la vértebra, estabilizando la fractura y evitando el mayor colapso vertebral. Afortunadamente, las complicaciones graves de estos procedimientos, como el escape del cemento al canal raquídeo o la embolia pulmonar de cemento, son altamente infrecuentes. ${ }^{43}$ Estas técnicas pueden combinarse con radioterapia para hacer control local de la metástasis, sin afectar la eficacia de esta última. ${ }^{44}$

En los pacientes que tienen una fractura patológica o una lesión metastásica vertebral mecánicamente inestable (SINS alto) y sin compromiso neurológico, nosotros optamos por realizar técnicas de fijación vertebral percutánea. Estas son técnicas poco agresivas que permiten estabilizar eficazmente el segmento espinal comprometido, y disminuyen significativamente los riesgos de infección en comparación con la cirugía convencional de fijación de columna abierta. Luego de la estabilización percutánea, los pacientes pueden ser sometidos a radioterapia coadyuvante en forma precoz, sin mayores riesgos de complicaciones de heridas operatorias. ${ }^{45-47}$

En los pacientes con una metástasis vertebral con compromiso neurológico, ya sea por colapso vertebral o por invasión tumoral epidural y resistente a la radioterapia convencional, la recomendación de la literatura es la descompresión epidural, la estabilización quirúrgica, y la radioterapia de alta intensidad postoperatoria. ${ }^{33,48} \mathrm{La}$ cirugía convencional de descompresión epidural y fijación abierta, si bien es efectiva en mejorar el estatus neurológico, tiene altas tasas de complicaciones, que oscilan de un $25 \%$ a un $40 \%$, y que, a pesar de mejorar la función neurológica, pueden empeorar drásticamente la calidad de los últimos meses de vida del paciente. ${ }^{45}$ Siguiendo en la línea de utilizar técnicas de menor agresión, en estos pacientes, nosotros hemos optado por el uso de fijación vertebral y descompresión epidural miniinvasiva. La descompresión neural se hace por vía transmuscular, alejada de la línea media, realizando una resección intralesional y reduciendo así la masa tumoral alrededor de las estructuras neurales, lo que en la actualidad se conoce como "cirugía de separación tumoral". Esto crea un espacio entre el tumor y el saco dural, de manera que la radioterapia postoperatoria estereotáxica pueda ser aplicada efectivamente sobre el tumor remanente con un espacio de seguridad que evite dañar las estructuras 
neurales. La asociación de técnicas miniinvasivas, adecuadamente aplicadas para evitar descompresión insuficiente con radioterapia estereotáxica postoperatoria, permite combinar las ventajas de una fijación vertebral y de una descompresión neural de menor riesgo quirúrgico con una radioterapia de alta concentración, logrando un efectivo control local, con tasas de recurrencia menores al 5\% al año ${ }^{48-50}$ (- Figura 2). A nuestro juicio, la cirugía miniinvasiva de las metástasis vertebrales puede beneficiar a pacientes con pronóstico de vida más limitado o con mayor riesgo de complicaciones.

\section{Conclusiones}

En los pacientes con metástasis vertebral, el tratamiento es principalmente paliativo. El avance en los tratamientos oncológicos y adyuvantes, junto al desarrollo tecnológico de implantes y técnicas quirúrgicas menos invasivas, ha permitido aumentar la sobrevida, con una menor tasa de complicaciones asociada. Esto permite cumplir el objetivo principal en el manejo de estos pacientes: mejorar su calidad de vida en su última etapa.

El marco de decisión NOMS nos da una visión amplia del paciente y su enfermedad metastásica vertebral, y permite que enfoquemos nuestros tratamientos según su estatus neurológico, biología tumoral, estabilidad espinal, y su estado general.

Una comunicación fluida entre oncólogos, radioterapeutas y cirujanos de columna permite consolidar este marco de enfrentamiento, y nos permite ofrecer a los pacientes con cáncer avanzado soluciones personalizadas, oportunas, eficaces y con la menor morbilidad posible.

\section{Conflicto de Intereses}

Los autores declaran que no hay conflicto de intereses.

\section{Referencias}

1 Joaquim AF, Powers A, Laufer I, Bilsky MH. An update in the management of spinal metastases. Arq Neuropsiquiatr 2015;73 (09):795-802

2 Klimo P Jr, Schmidt MH. Surgical management of spinal metastases. Oncologist 2004;9(02):188-196

3 Falkner U. Järhult J, Wersäll P, Cavallin-Ståhl E. A systematic overview of radiation therapy effects in skeletal metastases. Acta Oncol (Madr) 2003;42(5-6):620-633

4 Bilsky M, Smith M. Surgical approach to epidural spinal cord compression. Hematol Oncol Clin North Am 2006;20(06): 1307-1317

5 Siegel RL, Miller KD, Jemal A. Cancer statistics, 2015. CA Cancer J Clin 2015;65(01):5-29

6 Perou CM, Sørlie T, Eisen MB, et al. Molecular portraits of human breast tumours. Nature 2000;406(6797):747-752

7 Lee IH. Molecular Prognostic and Predictive Assays in Breast Cancer: A Practical Review. AJSP: Reviews \& Reports 2016;21 (01):4-10

8 Padhy LC, Shih C, Cowing D, Finkelstein R, Weinberg RA. Identification of a phosphoprotein specifically induced by the transforming DNA of rat neuroblastomas. Cell 1982;28(04): 865-871

9 Piccart-Gebhart MJ, Procter M, Leyland-Jones B, et al; Herceptin Adjuvant (HERA) Trial Study Team. Trastuzumab after adjuvant chemotherapy in HER2-positive breast cancer. N Engl J Med 2005; 353(16):1659-1672

10 Slamon DJ, Leyland-Jones B, Shak S, et al. Use of chemotherapy plus a monoclonal antibody against HER2 for metastatic breast cancer that overexpresses HER2. N Engl J Med 2001;344(11): 783-792

11 Swain SM, Baselga J, Kim SB, et al; CLEOPATRA Study Group. Pertuzumab, trastuzumab, and docetaxel in HER2-positive metastatic breast cancer. N Engl J Med 2015;372(08):724-734

12 Verma S, Miles D, Gianni L, et al; EMILIA Study Group. Trastuzumab emtansine for HER2-positive advanced breast cancer. N Engl J Med 2012;367(19):1783-1791

13 Geyer CE, Forster J, Lindquist D, et al. Lapatinib plus capecitabine for HER2-positive advanced breast cancer. N Engl J Med 2006;355 (26):2733-2743

14 Davies C, Godwin J, Gray R, et al; Early Breast Cancer Trialists' Collaborative Group (EBCTCG) Relevance of breast cancer hormone receptors and other factors to the efficacy of adjuvant tamoxifen: patient-level meta-analysis of randomised trials. Lancet 2011;378(9793):771-784

15 Chia SK, Speers CH, D'yachkova Y, et al. The impact of new chemotherapeutic and hormone agents on survival in a population-based cohort of women with metastatic breast cancer. Cancer 2007; 110(05):973-979

16 Cristofanilli M, Turner NC, Bondarenko I, et al. Fulvestrant plus palbociclib versus fulvestrant plus placebo for treatment of hormone-receptor-positive, HER2-negative metastatic breast cancer that progressed on previous endocrine therapy (PALOMA-3): final analysis of the multicentre, double-blind, phase 3 randomised controlled trial. Lancet Oncol 2016;17(04): 425-439

17 Siegel RL, Miller KD, Jemal A. Cancer statistics, 2016. CA Cancer J Clin 2016;66(01):7-30

18 NSCLC Meta-Analyses Collaborative Group. Chemotherapy in addition to supportive care improves survival in advanced nonsmall-cell lung cancer: a systematic review and meta-analysis of individual patient data from 16 randomized controlled trials. J Clin Oncol 2008;26(28):4617-4625

19 Lee CK, Brown C, Gralla RJ, et al. Impact of EGFR inhibitor in nonsmall cell lung cancer on progression-free and overall survival: a meta-analysis. J Natl Cancer Inst 2013;105(09):595-605

20 Brahmer J, Reckamp KL, Baas P, et al. Nivolumab versus Docetaxel in Advanced Squamous-Cell Non-Small-Cell Lung Cancer. N Engl J Med 2015;373(02):123-135

21 Borghaei H, Paz-Ares L, Horn L, et al. Nivolumab versus Docetaxel in Advanced Nonsquamous Non-Small-Cell Lung Cancer. N Engl J Med 2015;373(17):1627-1639

22 Herbst RS, Baas P, Kim DW, et al. Pembrolizumab versus docetaxel for previously treated, PD-L1-positive, advanced non-small-cell lung cancer (KEYNOTE-010): a randomised controlled trial. Lancet 2016;387(10027):1540-1550

23 Antonia SJ, Villegas A, Daniel D, et al; PACIFIC Investigators. Durvalumab after Chemoradiotherapy in Stage III Non-SmallCell Lung Cancer. N Engl J Med 2017;377(20):1919-1929

24 Oefelein MG, Feng A, Scolieri MJ, Ricchiutti D, Resnick MI. Reassessment of the definition of castrate levels of testosterone: implications for clinical decision making. Urology 2000;56(06):1021-1024

25 Seidenfeld J, Samson DJ, Hasselblad V, et al. Single-therapy androgen suppression in men with advanced prostate cancer: a systematic review and meta-analysis. Ann Intern Med 2000;132 (07):566-577

26 Crawford ED, Eisenberger MA, McLeod DG, et al. A controlled trial of leuprolide with and without flutamide in prostatic carcinoma. N Engl J Med 1989;321(07):419-424

27 Scher HI, Fizazi K, Saad F, et al; AFFIRM Investigators. Increased survival with enzalutamide in prostate cancer after chemotherapy. N Engl J Med 2012;367(13):1187-1197 
28 de Bono JS, Logothetis CJ, Molina A, et al; COU-AA-301 Investigators. Abiraterone and increased survival in metastatic prostate cancer. N Engl J Med 2011;364(21):1995-2005

29 Berthold DR, Pond GR, Soban F, de Wit R, Eisenberger M, Tannock IF. Docetaxel plus prednisone or mitoxantrone plus prednisone for advanced prostate cancer: updated survival in the TAX 327 study. J Clin Oncol 2008;26(02):242-245

30 de Bono JS, Oudard S, Ozguroglu M, et al; TROPIC Investigators. Prednisone plus cabazitaxel or mitoxantrone for metastatic castration-resistant prostate cancer progressing after docetaxel treatment: a randomised open-label trial. Lancet 2010;376 (9747):1147-1154

31 Sweeney CJ, Chen YH, Carducci M, et al. Chemohormonal Therapy in Metastatic Hormone-Sensitive Prostate Cancer. N Engl J Med 2015;373(08):737-746

32 Fizazi K, Tran N, Fein L, et al; LATITUDE Investigators. Abiraterone plus Prednisone in Metastatic, Castration-Sensitive Prostate Cancer. N Engl J Med 2017;377(04):352-360

33 Laufer I, Rubin DG, Lis E, et al. The NOMS framework: approach to the treatment of spinal metastatic tumors. Oncologist 2013;18 (06):744-751

34 Hortobagyi GN, Theriault RL, Porter L, et al. Efficacy of pamidronate in reducing skeletal complications in patients with breast cancer and lytic bone metastases. Protocol 19 Aredia Breast Cancer Study Group. N Engl J Med 1996;335(24): 1785-1791

35 Kohno N, Aogi K, Minami H, et al. Zoledronic acid significantly reduces skeletal complications compared with placebo in Japanese women with bone metastases from breast cancer: a randomized, placebo-controlled trial. J Clin Oncol 2005;23(15): 3314-3321

36 Stopeck AT, Lipton A, Body JJ, et al. Denosumab compared with zoledronic acid for the treatment of bone metastases in patients with advanced breast cancer: a randomized, double-blind study. J Clin Oncol 2010;28(35):5132-5139

37 Wong MH, Stockler MR, Pavlakis N. Bisphosphonates and other bone agents for breast cancer. Cochrane Database Syst Rev 2012; (02):CD003474. Doi: 10.1002/14651858.cd003474.pub3

38 Bilsky MH, Laufer I, Fourney DR, et al. Reliability analysis of the epidural spinal cord compression scale. J Neurosurg Spine 2010; 13(03):324-328

39 Fisher CG, DiPaola CP, Ryken TC, et al. A novel classification system for spinal instability in neoplastic disease: an evidence-based approach and expert consensus from the Spine Oncology Study Group. Spine 2010;35(22):E1221-E1229

40 Bate BG, Khan NR, Kimball BY, Gabrick K, Weaver J. Stereotactic radiosurgery for spinal metastases with or without separation surgery. J Neurosurg Spine 2015;22(04):409-415

41 Lo SS, Sahgal A, Chang EL. Major Complications Associated with Stereotactic Ablative Radiotherapy for Spinal Metastasis. In: AOSPine Masters Series Metastatic Spinal Tumors. 1st Ed. New York: Thieme Medical Publishers, Inc.; 2015:23-33

42 Patchell RA, Tibbs PA, Regine WF, et al. Direct decompressive surgical resection in the treatment of spinal cord compression caused by metastatic cancer: a randomised trial. Lancet 2005;366 (9486):643-648

43 Hulme PA, Krebs J, Ferguson SJ, Berlemann U. Vertebroplasty and kyphoplasty: a systematic review of 69 clinical studies. Spine 2006;31(17):1983-2001

44 Kassamali RH, Ganeshan A, Hoey ETD, Crowe PM, Douis H, Henderson J. Pain management in spinal metastases: the role of percutaneous vertebral augmentation. Ann Oncol 2011;22(04): 782-786

45 Wise JJ, Fischgrund JS, Herkowitz HN, Montgomery D, Kurz LT. Complication, survival rates, and risk factors of surgery for metastatic disease of the spine. Spine 1999;24(18):1943-1951

46 Lee RS, Batke J, Weir L, Dea N, Fisher CG. Timing of surgery and radiotherapy in the management of metastatic spine disease: expert opinion. J Spine Surg 2018;4(02):368-373

47 Barzilai O, McLaughlin L, Amato MK, et al. Minimal Access Surgery for Spinal Metastases: Prospective Evaluation of a Treatment Algorithm Using Patient-Reported Outcomes. World Neurosurg 2018;120:e889-e901

48 Laufer I, Iorgulescu JB, Chapman T, et al. Local disease control for spinal metastases following "separation surgery" and adjuvant hypofractionated or high-dose single-fraction stereotactic radiosurgery: outcome analysis in 186 patients. J Neurosurg Spine 2013;18(03):207-214

49 Schwab JH, Gasbarrini A, Cappuccio M, et al. Minimally Invasive Posterior Stabilization Improved Ambulation and Pain Scores in Patients with Plasmacytomas and/or Metastases of the Spine. Int J Surg Oncol 2011;2011:239230. Doi: 10.1155/2011/239230

50 Donnelly DJ, Abd-El-Barr MM, Lu Y. Minimally Invasive Muscle Sparing Posterior-Only Approach for Lumbar Circumferential Decompression and Stabilization to Treat Spine MetastasisTechnical Report. World Neurosurg 2015;84(05):1484-1490 